\title{
Method for fabricating a low stress x-ray mask using annealable amorphous refractory compounds
}

\author{
W. J. Dauksher, D. J. Resnick, K. D. Cummings, and J. Baker \\ Phoenix Corporate Research Laboratories, Motorola, Tempe, Arizona 85284
}

R. B. Gregory and N. D. Theodore

Materials Characterization Laboratory, Motorola, Mesa, Arizona 85202

J. A. Chan

MOS-12, Motorola, Chandler, Arizona 85224

W. A. Johnson and C. J. Mogab

APRDL, Motorola, Austin, Texas 78721

M.-A. Nicolet and J. S. Reid

California Institute of Technology, Pasadena, California 91125

(Received 2 June 1995; accepted 2 August 1995)

\begin{abstract}
X-ray masks have been fabricated by depositing a compressively stressed refractory material on a wafer, annealing to a zero stress state, and then forming the membrane. Amorphous TaSiN and TaSi alloys deposited with a magnetron sputter tool have been extensively characterized in terms of resistivity, composition, defectivity, surface roughness, and crystalline state. Optimization in terms of these parameters has resulted in base line selection of absorber films of the following compositions: $\mathrm{Ta}_{61} \mathrm{Si}_{17} \mathrm{~N}_{21}$ and $\mathrm{Ta}_{75} \mathrm{Si}_{23}$. The process is shown to be extendable to an entire class of amorphous annealable refractory materials. Careful studies of deposition and annealing conditions have resulted in a $4 \times$ reduction of image placement to the $30 \mathrm{~nm}$ maximum vector level. Finally, the importance of stress gradients is experimentally verified. () 1995 American Vacuum Society.
\end{abstract}

\section{INTRODUCTION}

At least in the United States, conventional x-ray mask fabrication typically involves a membrane formation sequence followed by an absorber definition process. Recently, it has been demonstrated that the deposition of a refractory material on a membrane may result in a radial stress gradient due to temperature variations across the membrane. ${ }^{1,2}$ The resulting stress gradient has been shown to induce unacceptable image placement errors on the mask.

One way to overcome this problem is to deposit a refractory material on a wafer, anneal the material to a zero stress state, and then form the membrane. In order to implement this process, a suitable absorber must be identified. While the process appears to be extendable to a whole class of refractory based compounds, ${ }^{3-5}$ amorphous $\mathrm{TaSiN}$ and $\mathrm{TaSi}$ films, ${ }^{6,7}$ deposited in a direct current (dc) magnetron sputter system, have been shown to satisfy the process criteria. The materials are extremely compressive as deposited, but are easily annealed to zero stress at a temperature of about $470{ }^{\circ} \mathrm{C}$. Stress control is achieved via an iterative anneal and stress measurement sequence.

Other characteristics of the TaSiN and TaSi alloys make them attractive as $\mathrm{x}$-ray absorber materials. Once annealed, the films are stable and experience no change in stress during subsequent processing steps. Surface roughness, which is an important issue for mask inspection purposes, has been shown to be very low, on the order of $50 \AA$ peak to valley. While the films are readily patterned with conventional dry etch processes, they possess a resistance to chemical attack that lends itself to aggressive wet cleans for defect reduction purposes. ${ }^{8}$ In addition, because TaSiN and TaSi films are mostly Ta by weight, the alloys have a sufficient density so that suitable mask contrast is attainable without taxing feature aspect ratio. Finally, the amorphous state of the films has been shown to be advantageous, notably in terms of focused ion beam (FIB) repair. ${ }^{9}$

This paper will describe the process necessary to fabricate an x-ray mask and discuss the characteristics of the TaSiN films. Initial in-plane distortion data on pattern transferred TaSiN and TaSi masks will also be presented.

\section{EXPERIMENTAL METHODS}

The initial identification of TaSiN deposition parameter space was accomplished by running a mixed level, full factorial design of experimental study. The input factors varied were power, pressure, and $\% \mathrm{~N}_{2}$ in Ar. The films were dc magnetron sputtered in an MRC 603 deposition tool after performing a 2 min target presputter. Nominal film thickness was $5000 \AA$, and adjustments were made to pallet scan speed accordingly. Film thickness was measured by taking profilometer scans across an abrupt knife edge created by masking an area during the deposition process.

The film properties studied for optimization included resistivity, composition, defectivity, surface roughness, and crystalline state. TaSiN resistivity was measured with a four point probe on films deposited over an oxide insulating layer. Compositional analysis of TaSiN was accomplished using Rutherford backscattering spectrometry (RBS) which provides measurements of elemental areal densities from first principles. The $\mathrm{He}^{2+}$ ions at $1892 \mathrm{keV}$ were normally incident on the sample. Backscattered ions were detected at $170^{\circ}$ relative to the incident beam. Defectivity data obtained for 
the various deposition runs was limited to optical inspection. Surface roughness was checked with an atomic force microscope (AFM) operating in the tapping mode over 1 and 25 $\mu \mathrm{m}^{2}$ scan areas. Finally, the crystalline state was studied via a JEOL JEM 200CX transmission electron microscope, operating at $200 \mathrm{kV}$. Plan-view transmission electron microscopy (PTEM) specimens were prepared in a $\langle 001\rangle$ substrate geometry. These were then analyzed using bright-field and dark-field imaging under varying conditions of electronbeam tilt.

The basic x-ray mask fabrication sequence consisted of depositing TaSiN on a wafer, annealing the stress to a zero average stress state, and then forming the mask blank. Typically, a two step rapid thermal annealing (RTA) process was employed: a coarse anneal followed by stress measurement for selecting the fine tuning anneal temperature. Stress measurements were taken using a Tencor FLX-5200h laser deflection radius of curvature type tool.

All of the masks fabricated were $40 \mathrm{~mm}$ in diameter with silicon nitride as the membrane material. Patterns were written in Shipley SNR200 resist using a Cambridge EBML 300 $40 \mathrm{keV}$ e-beam tool. Pattern transfer was accomplished in a loadlock, dual chamber, backside helium cooled Plasma Therm 770 series etcher with reactive ion etching (RIE) and electron cyclotron resonance (ECR) etch capability.

Distortion of the X-ray masks by stress and stress gradients was studied by measuring image placement of fiducial arrays before and after TaSiN etch. A Nikon $X Y 3 i$ image placement tool was used to measure the position of the crosses. The fiducial patterns were always written in negative resist so that subsequent removal of most of the metal from the mask would exacerbate the effect of any stress or stress gradient present in the metal.

\section{MATERIALS CHARACTERIZATION RESULTS}

Compositional film studies indicated that higher sputter power and lower pressure tended to increase tantalum incor-

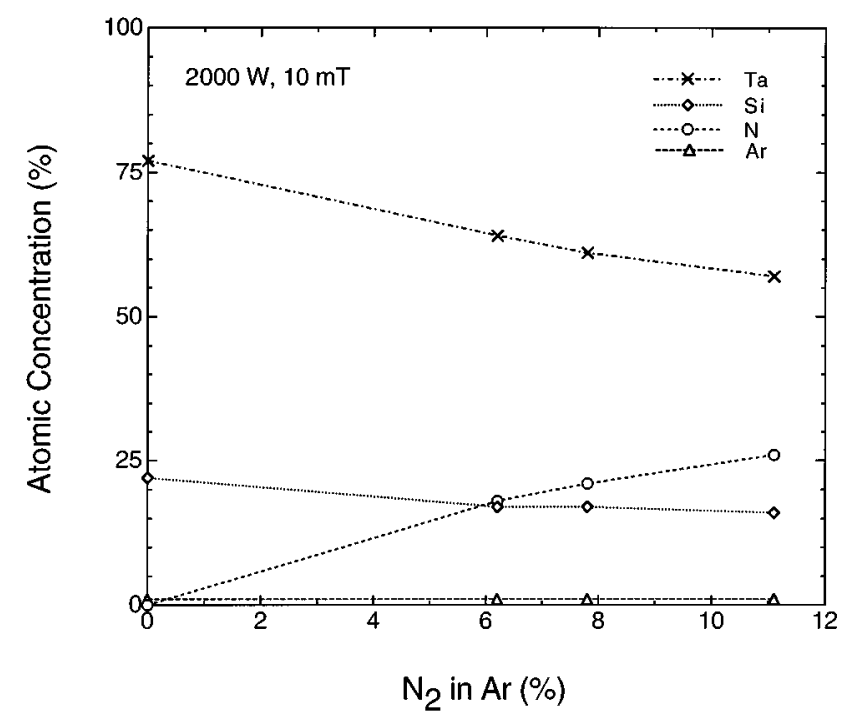

FIG. 1. Plot of film composition vs $\% \mathrm{~N}_{2}$ in Ar for films sputtered at $2000 \mathrm{~W}$ and $10 \mathrm{mT}$. Composition was determined via RBS.

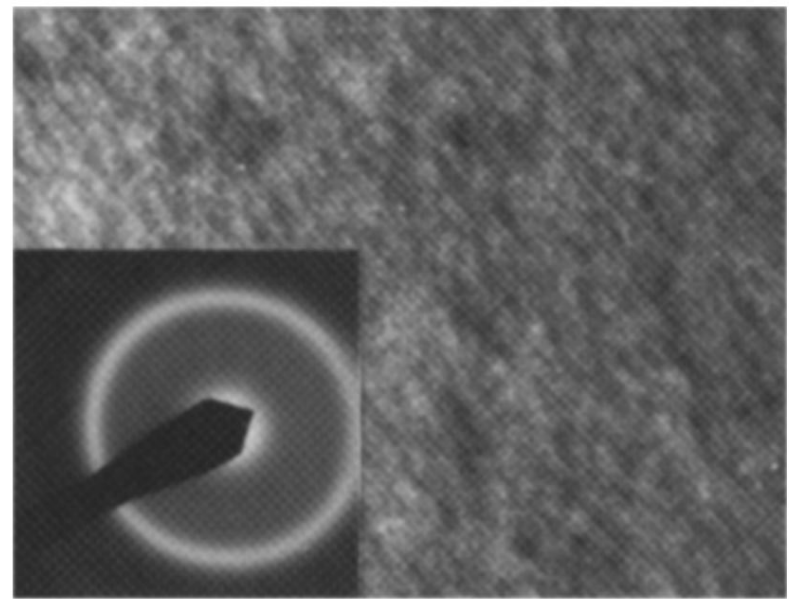

FIG. 2. TEM dark-field image of a TaSiN film. No evidence of a nanocrystalline phase is present. The inlaid diffraction pattern indicates an amorphous film, with some degree of short range order.

poration in the films, primarily at the expense of nitrogen. In order to maximize film density, nominal sputter conditions of $2000 \mathrm{~W}$ and $10 \mathrm{mT}$ were selected from among those studied. Figure 1 contains a plot of atomic concentration versus $\% \mathrm{~N}_{2}$ in Ar for the films deposited under these conditions. It can be seen that approximately 1 at. $\%$ of the films is incorporated Ar. Initial mask fabrication was carried out using reactively sputtered films of the following composition: $\mathrm{Ta}_{61} \mathrm{Si}_{17} \mathrm{~N}_{21}$ (93.5 w/o Ta, 4.0 w/o Si, 2.5 w/o N). Subsequent studies involved TaSi films sputtered in pure Ar. These films were analytically determined to be $\mathrm{Ta}_{75} \mathrm{Si}_{23}$ (95.5 w/o Ta, $4.5 \mathrm{w} / \mathrm{o}$ $\mathrm{Si}$ ). To a first approximation, it was found that the resistivity tracked well with nitrogen concentration in the films. The resistivity of the TaSiN base line material was about 210 $\mu \Omega \mathrm{cm}$; that of the base line TaSi film was $150 \mu \Omega \mathrm{cm}$.

In general, all of the TaSiN and TaSi films deposited were found to be amorphous. Figure 2 is a representative TEM photograph of a dark-field image obtained from a base line $\mathrm{TaSiN}$ film. No evidence of a nanocrystalline phase exists. In

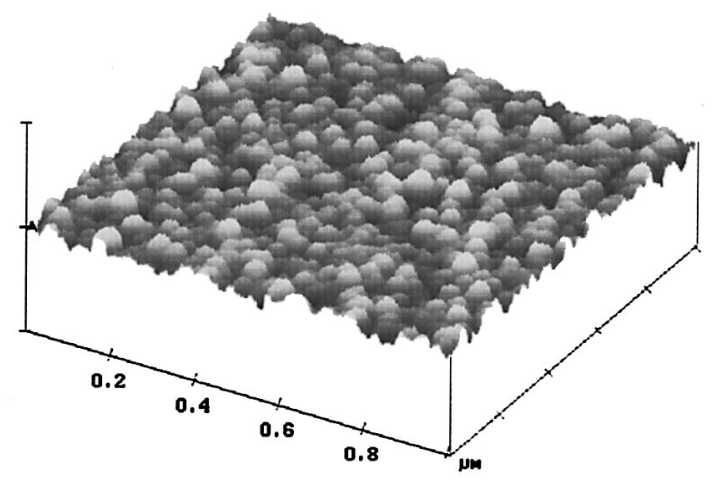

TaSiN

X $0.200 \mathrm{Mm} / \mathrm{dil}$

FIg. 3. AFM image of a $5000 \AA$ TaSiN film. Peak to valley surface roughness is about $50 \AA$. 


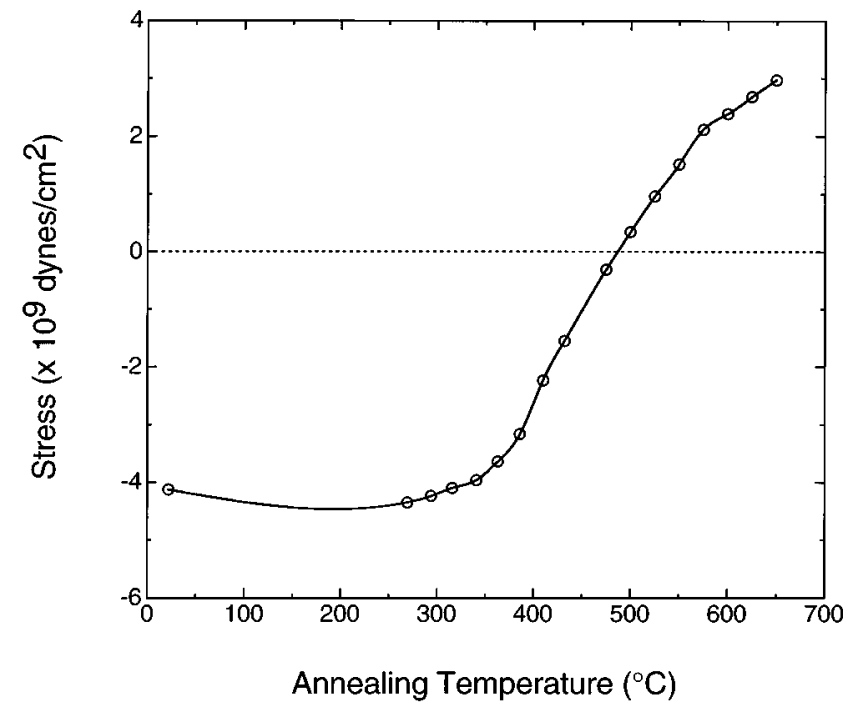

FIG. 4. Graph of stress vs annealing temperature for TaSiN films deposited on wafers. A zero stress film is obtained at about $470{ }^{\circ} \mathrm{C}$. The stress has been shown to be stable at temperatures below the annealing temperature.

addition, the inlaid diffraction pattern in Fig. 2 is indicative of an amorphous film with some short range order present. In fact, films with nitrogen concentrations below about 20 at. \% were found to have some short range order present. Conversely, a nanocrystalline phase seemed to exist at high $(>50$ at. \%) nitrogen concentrations.

Optical inspection of the films deposited did not reveal the presence of any spits, inhomogeneities, or other defects that might be associated with a sputtering process. Peak to valley surface roughness was found to be about $50 \AA$ ( $5 \AA$ rms surface roughness) for both $\mathrm{TaSiN}$ and $\mathrm{TaSi}$ films. An AFM image showing surface texture and roughness is shown in Fig. 3. For comparison purposes, these roughness numbers

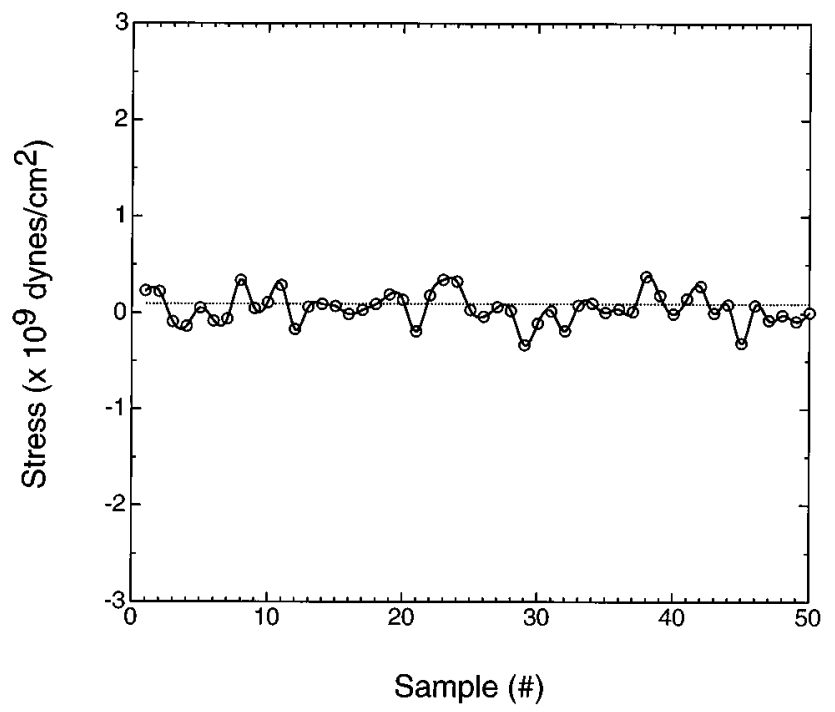

FIG. 5. Stress is plotted for 50 consecutive samples after employing an experimental two step annealing sequence to reduce the as-deposited $\mathrm{TaSiN}$ stress. Average stress for 50 samples was $5.0 \times 10^{7} \mathrm{dyn} / \mathrm{cm}^{2} \pm 1.6 \times 10^{8}$ $\mathrm{dyn} / \mathrm{cm}^{2}$.

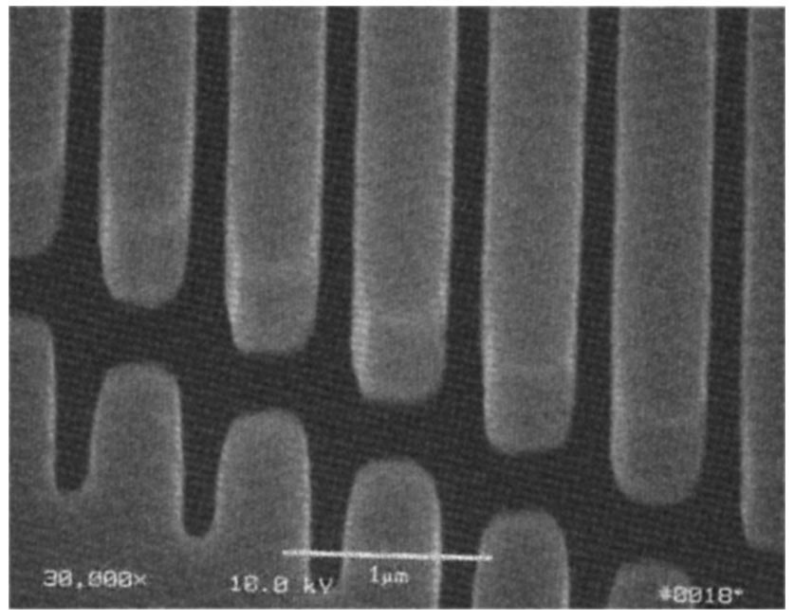

FIG. 6. Clustered $0.45 \mu \mathrm{m}$ lines/0.15 $\mu \mathrm{m}$ spaces in $5000 \AA$ of TaSiN. Note the lack of apparent grain structure in the absorber.

are about $1 / 7$ of that observed for fine grained plated gold absorber films. ${ }^{10}$

\section{MASK FABRICATION RESULTS}

In order to avoid issues associated with tailoring sputtering conditions to deposit a low stress film or depositing on membranes with temperature homogenization and feedback loops, it was decided to deposit a refractory material on a wafer, anneal to a low stress state, and then form a mask blank. A plot of stress versus annealing temperature for TaSiN is shown in Fig. 4. The material is extremely compressive as deposited, on the order of $-4.0 \times 10^{9} \mathrm{dyn} / \mathrm{cm}^{2}$ $(-400 \mathrm{MPa})$. Although initial material relaxation is not linear, above about $400{ }^{\circ} \mathrm{C}$ relaxation follows a predictable, linear behavior. For the case of the base line TaSiN material, the

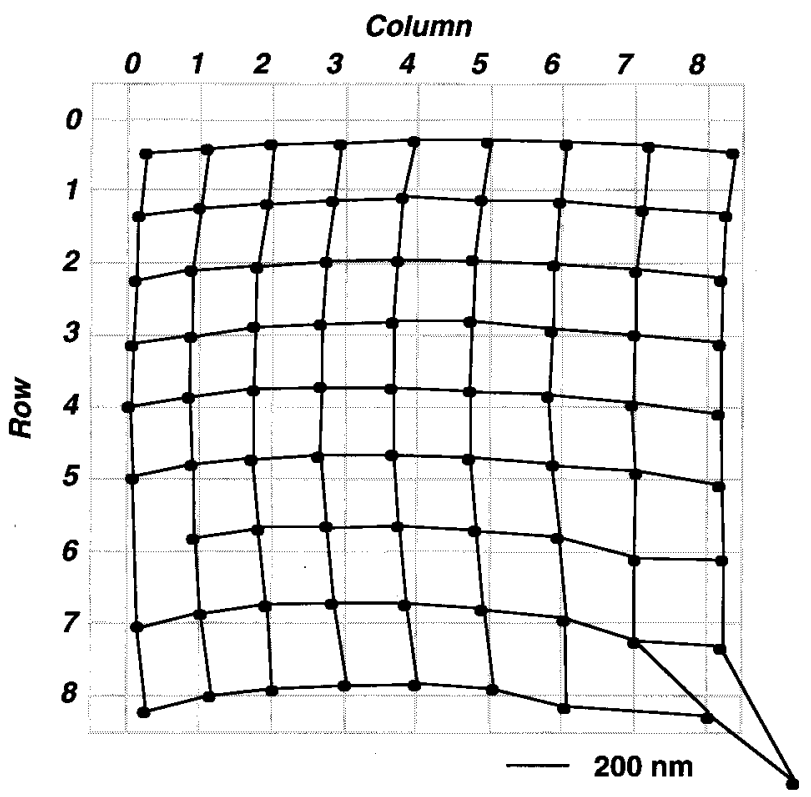

FIG. 7. Nikon $X Y 3 i$ image placement plot of a standard TaSiN film before and after etch of the 5000- $\AA$-thick metal layer. Maximum deviations are about $90 \mathrm{~nm}$ in $x$ and $120 \mathrm{~nm}$ in $y$, excluding fliers. 


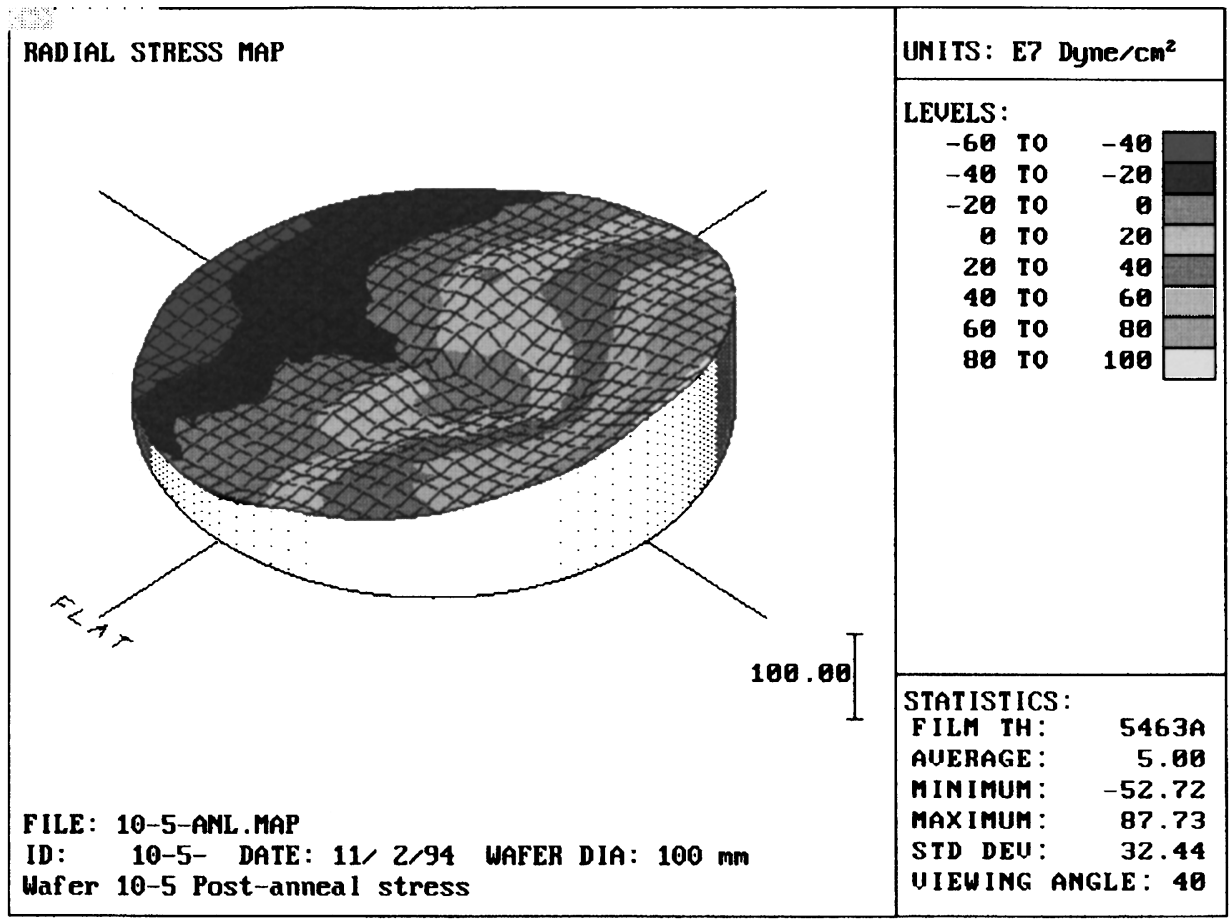

FIG. 8. Tencor FLX-5200h 3D stress map after deposition and anneal of $5000 \AA \mathrm{TaSiN}$ on a 4 in. wafer. A stress gradient of about $1.4 \times 10^{9}$ dyn/ $\mathrm{cm}^{2}$ exists across the wafer.

relaxation rate around the zero stress crossover point is about $1 \times 10^{8} \mathrm{dyn} / \mathrm{cm}^{2}$ for every $4{ }^{\circ} \mathrm{C}$ temperature increase. After film deposition, stress is measured, the film is annealed to the linear relaxation regime, and the stress is remeasured. Knowing the change of stress with temperature allows for a trivial second anneal temperature to be calculated. For the case of $\mathrm{TaSiN}$, the zero stress point is at a temperature of about $470{ }^{\circ} \mathrm{C}$.

Initial annealing studies to determine feasibility targeted achieving stress control within the arbitrary range of

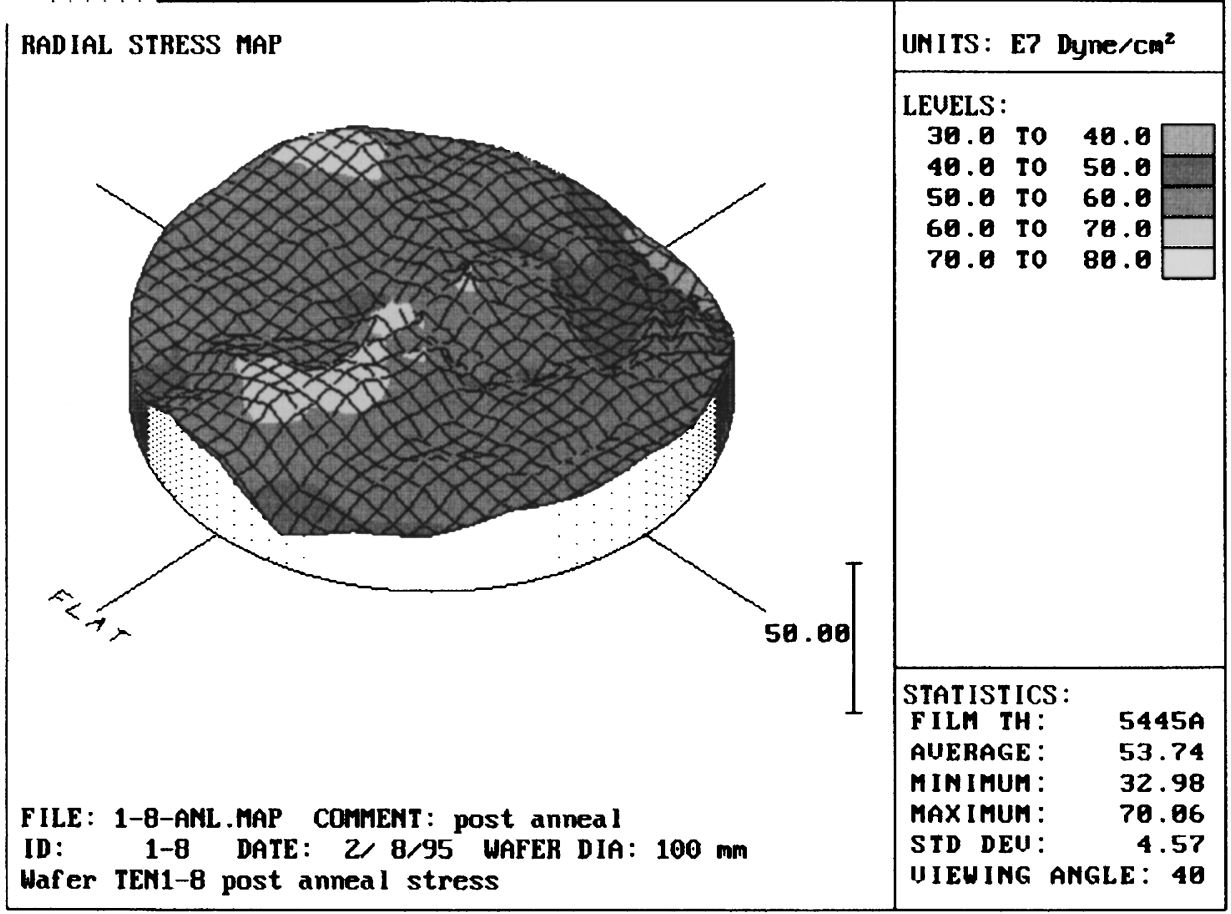

FIG. 9. Tencor FLX-5200h 3D stress map after modification of the deposition and anneal conditions to reduce stress gradients. Stress variation across the wafer is now on the order of $3.8 \times 10^{8} \mathrm{dyn} / \mathrm{cm}^{2}$. 


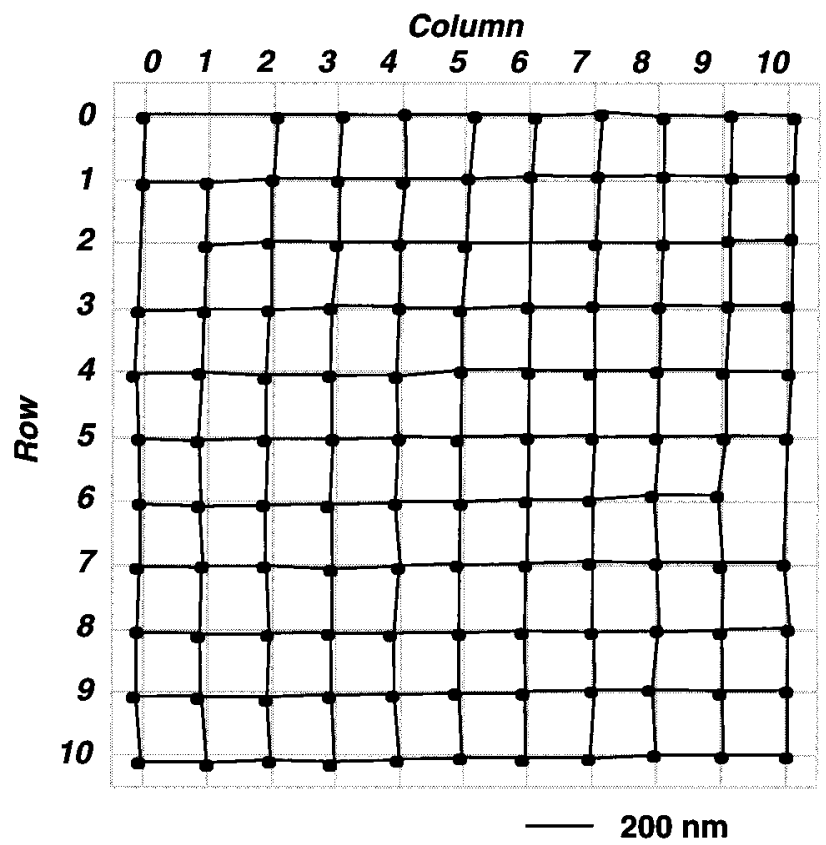

FIG. 10. Image placement of a $5000 \AA$ metal film after modification of the deposition and annealing conditions. Maximum deviations in $x$ and $y$ are 32 and $22 \mathrm{~nm}$, respectively.

$\pm 2.0 \times 10^{8} \mathrm{dyn} / \mathrm{cm}^{2}$. Figure 5 demonstrates the stress control observed for the first 50 consecutive samples after employing an experimental two step RTA annealing sequence. The average stress for these samples was found to be $5.0 \times 10^{7}$ $\mathrm{dyn} / \mathrm{cm}^{2} \pm 1.6 \times 10^{8} \mathrm{dyn} / \mathrm{cm}^{2}$. It can be seen that approximately $80 \%$ of these samples had final stresses within the target range. After learning from these initial samples and making improvements to the annealing process, subsequent lots have been successfully annealed to tighter standard deviations, near the lower limit of the radius of curvature technique employed $\left(\sim 1.0 \times 10^{8} \mathrm{dyn} / \mathrm{cm}^{2}\right.$, given current film and substrate thicknesses). It is believed that the sensitivity of the technique can be improved further so that manufacturing issues of stress control are not a concern.

The refractory deposition on a wafer/two step annealing process described above has additional advantages as well. At temperatures below the annealing point, the stress of the refractory material is stable. Subsequent processing operations, such as anodic bonding $\left(\sim 350^{\circ} \mathrm{C}\right)$, will not adversely impact film stress. Other film properties, such as the film composition, surface roughness, and amorphous state were all found to be invariant after the annealing process. Furthermore, the process should be extendable to a whole class of refractory based compounds.

An example of features attainable on a completed mask is shown in Fig. 6. The amorphous nature of the TaSiN and the robustness of the pattern transfer process are reflected in the fidelity of the etched $0.45 \mu \mathrm{m}$ lines and $0.15 \mu \mathrm{m}$ spaces imaged in this micrograph.

\section{IMAGE PLACEMENT RESULTS}

Historically speaking, one of the motivations for pursuing the wafer deposition/anneal/membrane formation sequence described above was the belief that stress gradients in general, and radial stress gradients in particular, would not be present. Fabrication of masks proved otherwise. Figure 7 is a Nikon $X Y 3 i$ image placement plot of fiducial positions before and after TaSiN etch. Excluding fliers, maximum deviations are approximately $90 \mathrm{~nm}$ in $x$ and $120 \mathrm{~nm}$ in $y$. The fiducial spacing on this mask was on $3 \mathrm{~mm}$ centers. In order to verify the existence of a stress gradient, 3D stress plots of TaSiN films deposited on wafers were generated using a radius of curvature tool. Figure 8 shows a stress map of a base line $\mathrm{TaSiN}$ film deposited and annealed in the same fashion as that of the film in Fig. 7. It can be seen that a stress gradient on the order of $1.4 \times 10^{9} \mathrm{dyn} / \mathrm{cm}^{2}$ exists across the wafer. Stress gradients are in both axes, although more pronounced in one axis than the other.

Using the 3D wafer stress measurement technique as a screening tool, sources of stress nonuniformity were identified during the deposition and annealing processes. In this case, the relative simplicity of the sputter and annealing tools aided in identification of these sources. The principal contributors were found to be nonuniform heating of the wafers during pallet scanning (left to right) in the deposition process, the manner of gas flow (top to bottom) into the sputter chamber, hot spots in the RTA, and nonuniform gas flow around the RTA susceptor. Several deposition and annealing techniques were identified that proved promising for fabrication of reduced stress gradient masks.

The first of these entailed TaSi sputter deposition with wafer rotation and a modified RTA annealing process to address the sources of stress nonuniformity. In Fig. 9 is a 3D wafer stress plot of a film deposited and annealed in this manner. The stress gradient is reduced to about $3.8 \times 10^{8}$ $\mathrm{dyn} / \mathrm{cm}^{2}$ on the $4 \mathrm{in}$. wafer. Corresponding to this wafer data, Fig. 10 contains a Nikon $X Y 3 i$ image placement plot before and after TaSi etch. It can be seen that the maximum deviations are now about $32 \mathrm{~nm}$ in $x$ and $22 \mathrm{~nm}$ in $y$.

While there is still room for further improvement of these image placement numbers, several conclusions are obvious. To a first approximation, the use of a technique such as radius of curvature for stress gradient studies is valid. For the samples described above, a $4 \times$ reduction in stress gradient on the wafers resulted in about a $4 \times$ reduction in mask distortion. Improving the sensitivity of the technique might be necessary for further improvements, but this appears feasible. More importantly, though, this work serves to confirm the deleterious effects of stress gradients in films. Most work to date has been concerned with average stress effects and have ignored stress gradient effects. For a given mean film stress, a uniformly stressed film will cause a magnificationlike error on an x-ray mask, whereas a film with a stress gradient of the same magnitude will cause more significant distortion of the membrane. This is a consequence of boundary conditions: the absorber, or other film, is constrained at the edge of the membrane. Therefore, any stress variation across the absorber will impart a nonuniform force on the membrane itself, thereby causing more significant distortion. Very recent finite element modeling work done at the University of Wisconsin at Madison has also verified the impact of stress gradients. ${ }^{11}$ 


\section{CONCLUSIONS}

$\mathrm{TaSiN}$ and TaSi films have been proposed for incorporation as absorber layers on x-ray masks. Both films are amorphous over a very large operating regime. Surface roughness is on the order of $50 \AA$, peak to valley. Defect density, at least as determined via optical techniques, is very low. Since the Ta-based alloys are approximately $95 \%$ Ta by weight, the aspect ratio is not compromised significantly, as compared to pure Ta. The current wafer deposition, anneal, membrane formation sequence has demonstrated stress control of $1.0 \times 10^{8} \mathrm{dyn} / \mathrm{cm}^{2}$, near the limits of sensitivity of the laser deflection technique employed. By making improvements in sensitivity or by switching to another stress measurement technique, the process should be easily extendable for improved stress control. The importance of stress gradients cannot be understated. It is not sufficient that the average absorber film stress be zero; rather, the absorber stress must be low and uniform in order to avoid significant membrane distortion. Finally, image placement data was presented on $\mathrm{TaSi}$ films showing maximum deviations of about $32 \mathrm{~nm}$.

\section{ACKNOWLEDGMENTS}

The authors would like to recognize the efforts of Sherri Knight, Liz Velasquez, Cindy England, Mary Gonzalez, and Eddie Hernandez for assistance in fabrication of the x-ray masks. In addition, Diana Convey was always ready to provide timely AFM data. All RBS analytical work was per- formed at the Physics Department of the University of Arizona in Tucson under the auspices of John Leavitt, Lawrence McIntyre, and Donald Ashbaugh. Thanks are also due to Mark McCord of IBM for additional $100 \mathrm{keV}$ e-beam patterning and Chris Constantine of Plasma Therm for assistance in development of the refractory etch process. Finally, this work could not have been completed without the gracious help of Eric Fanucchi, Brian McCrossan, and Gina Corral of the Mesa Mask Shop with their Nikon XY $3 i$ tool.

${ }^{1}$ R. R. Kola et al., presented at the 38th International Symposium on Electron, Ion, and Photon Beams (unpublished, 1994).

${ }^{2}$ M. Mondol, H. Li, G. Owen, and H. I. Smith, J. Vac. Sci. Technol. B 12 , 4024 (1994).

${ }^{3}$ H. Yabe, K. Marumoto, K. Sasaki, S. Aya, K. Kise, and K. Nakamura, Proceedings of the 42nd Spring Meeting of the Japan Society of Applied Physics (1995) (to be published).

${ }^{4}$ T Shoki, R. Ohkubo, G. M. Wells, Y. Yamaguchi, K. Yamazaki, and F. Cerrina, Proceedings of Photomask Japan (1995) (to be published).

${ }^{5}$ T. Inoue, T. Kanayama, and M. Komuro, J. Vac. Sci. Technol. B 11, 2943 (1993).

${ }^{6}$ E. Kolawa, J. M. Molarius, C. W. Nieh, and M.-A. Nicolet, J. Vac. Sci. Technol. A 8, 3006 (1990).

${ }^{7}$ C.-K. Kwok, E. Kolawa, M.-A. Nicolet, and R. C. Lee, Mater. Res. Soc. Symp. Proc. 220, 259 (1991)

${ }^{8}$ I. Okada, T. Ohkubo, Y. Saitoh, M. Sekimoto, and T. Matsuda, Proc. SPIE (to be published).

${ }^{9}$ P. Blauner, J. Vac. Sci. Technol. B 13, 3070 (1995).

${ }^{10}$ W. J. Dauksher, D. J. Resnick, W. A. Johnson, and A. W. Yanof, Microelectron. Eng. 23, 235 (1994).

${ }^{11}$ M. Laudon and R. Engelstad (to be published). 